\title{
Competition between Traveling Fluid Waves of Left and Right Spiral Vortices and Their Different Amplitude Combinations
}

\author{
A. Pinter, M. Lücke, and Ch. Hoffmann \\ Institut für Theoretische Physik, \\ Universität des Saarlandes, \\ Postfach 151150, \\ D-66041 Saarbrücken, Germany
}

(Dated: October 1, 2018)

\begin{abstract}
Stability, bifurcation properties, and the spatiotemporal behavior of different nonlinear combination structures of spiral vortices in the counter rotating Taylor-Couette system are investigated by full numerical simulations and by coupled amplitude equation approximations. Stable crossspiral structures with continuously varying content of left and right spiral modes are found. They provide a stability transferring connection between the initially stable, axially counter propagating wave states of pure spirals and the axially standing waves of so-called ribbons that become stable slightly further away from onset of vortex flow.

PACS numbers: 47.20.-k, 47.54.+r, 47.32.-y, 47.10.+g
\end{abstract}


The combination of two counter propagating plane linear traveling waves (TWs) of equal amplitude creates a standing wave (SW). This property is also the reason for the possibility of SW structures in a variety of nonlinear pattern forming systems that show an oscillatory instability and inversion symmetry in one or more spatial directions [1]. Then two symmetry degenerate counter propagating TW structures as well as a nonlinear SW solution appear at the common oscillatory bifurcation threshold. However, in many systems like, e.g., binary mixture convection TW solutions bifurcate subcritically being initially amplitude unstable and then become strongly nonlinear on the stable upper solution branch. Moreover, the SW solution is often amplitude unstable and/or unstable against phase propagation and TW formation.

Thus, we consider here nonlinear combination states of left-spiral vortex (L-SPI) and right-spiral vortex (R-SPI) structures that — being mirror images of each other — travel either upwards or downwards in the annulus between two counter rotating cylinders of a Taylor-Couette setup [2, 3]. This system offers an easy experimental and numerical access ( $i$ ) to forwards bifurcating initially stable TWs (i.e., L-SPI and R-SPI), (ii) to forwards bifurcating SWs [called ribbons (RIBs) in the Taylor-Couette literature [2, 3]] that become stable slightly above threshold, and in particular also (iii) to stable cross-spirals (CR-SPI), i.e., combinations of L-SPI and R-SPI with different amplitudes which provide a stability transferring connection between TW and SW solution branches. This bifurcation scenario does not seem to have been reported so far [4].

Here, we describe stability, bifurcation properties, and the spatiotemporal behavior of the three oscillatory structures $(i)$-(iii) by two amplitude equations with a coupling that contains necessarily also a quintic order term to enable CR-SPI solutions. Their results are compared with numerical solutions of the full Navier-Stokes equations that we obtained for a system with radius ratio $\eta=1 / 2$ by methods described in [9].

The vortex structures $(i)$-(iii) are axially and azimuthally periodic with wave numbers $k=2 \pi / \lambda$ and $M$, respectively, with $\lambda=1.3$ and $M=2$ throughout this paper. They rotate with characteristic constant angular velocities into the same direction as the inner cylinder [9] and thereby they are forced to propagate axially except for RIB vortices which only rotate but do not propagate.

In order to characterize the spatiotemporal properties of the vortices we used also Fourier 
decompositions in azimuthal and axial direction

$$
f(r, \varphi, z, t)=\sum_{m, n} f_{m, n}(r, t) e^{i(m \varphi+n k z)} .
$$

For example, L-SPI (R-SPI) flow contains only modes with $m=n M(m=-n M)$ since it does not depend on $\varphi, z, t$ separately but only on the phase combination $\phi_{A}=M \varphi+k z-\omega_{A} t$ $\left(\phi_{B}=M \varphi-k z-\omega_{B} t\right)$. Here we use $A$ and $B$ to identify L-SPI and R-SPI properties, respectively. For SPI that are axial mirror images of each other the modes in (11) oscillate according to $f_{m, n}(r, t)=\delta_{m, \pm n M} e^{-i(m / M) \omega t} \tilde{f}_{ \pm n}(r)$ with a common frequency $\omega_{A}=\omega_{B}=$ $\omega(k, M)$. Thus, the color-coded plots in Fig. [(a,b) of the SPI in the $\varphi-z$ plane of an 'unrolled' cylindrical surface are patterns of straight stripes. They both move in positive $\varphi$-direction, i.e., into the direction of rotation of the inner cylinder [9] with angular phase velocity $\dot{\varphi}_{S P I}=\omega / M$. The L-SPI propagates upwards with axial phase velocity $w_{p h}^{A}=\omega / k$ and and its mirror image the R-SPI propagates downwards, $w_{p h}^{B}=-\omega / k$.

On the other hand, a CR-SPI is a kind of combination of a L-SPI and a R-SPI with different flow amplitudes and different frequencies. Thus, the CR-SPI spectrum contains two frequencies, say, $\omega_{S}$ and $\omega_{D}$, that are in general incommensurate. Within the amplitude equation approximation presented below $\omega_{S}=\left(\omega_{A}+\omega_{B}\right) / 2$ and $\omega_{D}=\left(\omega_{A}-\omega_{B}\right) / 2$. For the CR-SPI in Fig. 团(c) the amplitude of the L-SPI content is larger than the R-SPI content while $\omega_{A}<\omega_{B}$. Then the periodic deformation of the L-SPI-dominated stripe pattern in Fig. U(c) that is caused by the minority R-admixture propagates axially downwards with velocity $\omega_{D} / k$ while rotating with angular velocity $\omega_{S} / M$. The CR-SPI in Fig. I(d) is the mirror image of Fig. I(c) with reverse amplitude and frequency relations. For all CR-SPI investigated here $\omega_{S} \gg\left|\omega_{D}\right|$. RIB states are degenerate CR-SPI states with equal content of R-SPI and L-SPI contributions and $\omega_{D}=0$ which do not propagate axially.

Fig. [2] shows for two different $R_{1}$ bifurcation diagrams of SPI, CR-SPI, and RIB solutions versus $R_{2}$ and its reduced distance $\mu=\left(R_{2}-R_{2}^{0}\right) /\left|R_{2}^{0}\right|$ from the onset, $R_{2}^{0}$, of SPI and RIB flow. Here $R_{1}$ and $R_{2}$ are the Reynolds numbers defined by the rotational velocities of the inner and outer cylinder, respectively. Order parameters are the squared amplitudes $\left(|A|^{2},|B|^{2}\right)$ and frequencies $\left(\omega_{A}, \omega_{B}\right)$ of the dominant modes

$$
u_{2,1}(t)=|A| e^{-i \omega_{A} t} \quad, \quad u_{2,-1}(t)=|B| e^{-i \omega_{B} t}
$$

in the decomposition (11) of the radial velocity $u$ at mid-gap. For all vortex structures 
investigated here the moduli and frequencies of $u_{2, \pm 1}$ in Eq. (21) are constant.

The L-SPI $(A \neq 0, B=0)$ shown in Fig. 2 by circles and the RIB solution $(A=B)$ marked by diamonds bifurcate at $\mu=0$ with common linear frequency $\omega^{0}$ out of the unstructured CCF. However, with increasing $\mu$ their frequencies vary differently. The squared amplitudes of these two states grow basically linearly with $\mu$, albeit with different slopes.

Initially, the SPI is stable and the RIB is unstable. But then there appears at larger $\mu$ a stable CR-SPI solution (triangles) which transfers stability from the SPI to the RIB. The CR-SPI $(A \neq B)$ bifurcates with $B=0$ and finite $\omega_{B}$ out of the L-SPI as shown in Fig. 2 (bifurcation out of the symmetry degenerate R-SPI is analogous — just exchange $A$ and $B$ ). Then $|B|$ grows and $|A|$ decreases while $\omega_{A}$ increases and $\omega_{B}$ becomes smaller until the CR-SPI solution branches end with $A=B$ and $\omega_{A}=\omega_{B}$ in the RIB state. The latter loses stability outside the plot range of Fig. 2 to amplitude-modulated CR-SPI that are not discussed here.

Spatiotemporal properties, bifurcation, and stability behavior of the SPI, RIB, and CRSPI states close to onset can reasonably well be explained and described within an amplitudeequation approach. Therein the fields are represented by the superposition - here written down, e.g., for the radial velocity $u(r, \varphi, z, t)-$

$$
u=\left[A(t) \hat{u}_{A}(r) e^{i k z}+B(t) \hat{u}_{B}(r) e^{-i k z}\right] e^{i\left(M \varphi-\omega^{0} t\right)}+\text { c.c. }
$$

of just the linear critical modes $\hat{u}_{A, B}(r) e^{ \pm i k z} e^{i\left(M \varphi-\omega^{0} t\right)}$ of L- and R-SPI with slowly varying amplitudes $A$ and $B$, respectively. Here we normalize $\left|\hat{u}_{A}\right|$ and $\left|\hat{u}_{B}\right|$ to 1 at mid-gap. There the representation (3) is quite sufficient. But closer to the inner cylinder the vortex fields contain further axial and azimuthal modes that are generated via nonlinear interactions of the critical modes. However, it seems that close to onset they do not influence decisively the bifurcation and stability behavior that is governed by the modes retained in the approximation (3).

In order to reproduce the bifurcation and stability behavior of the aforementioned vortex states including the CR-SPI one needs coupled equations for $A$ and $B$ of at least quintic order [10]. Higher-order terms that are suggested in [3] are not necessary to ensure existence of CR-SPI solutions. Symmetry arguments [3] and simplicity considerations discussed below 
restrict the form of the equations to

$$
\begin{gathered}
\dot{A}=\left[a \mu+b|A|^{2}+c|B|^{2}+e\left(|A|^{2}-|B|^{2}\right)|B|^{2}\right] A \\
\dot{B}=\left[a \mu+b|B|^{2}+c|A|^{2}+e\left(|B|^{2}-|A|^{2}\right)|A|^{2}\right] B .
\end{gathered}
$$

The coefficients $a-e$ are complex but only the moduli $|A|$ and $|B|$ enter into the square brackets. We have determined all coefficients by comparing with bifurcation diagrams obtained for the flow at mid-gap position.

We are seeking solutions of (44) of the form $A(t)=|A| e^{-i \Omega_{A} t}, B(t)=|B| e^{-i \Omega_{B} t}$ with constant moduli and frequencies. Here $\Omega_{A, B}=\omega_{A, B}-\omega^{0}$ are the deviations of the frequencies from the critical ones according to (2, 3).

In view of Fig. 22 we have neglected here for simplicity reasons quintic contributions to SPI amplitudes and frequencies by discarding terms of the form $d|A|^{4} A$ and $d|B|^{4} B$ in (4a) and (4b), respectively. Taking $a=1+a_{i}$ one then has $|A|^{2}=\mu /\left(-b_{r}\right)$ and $\Omega_{A}=-a_{i} \mu-b_{i}|A|^{2}$ for, say, a L-SPI (for a R-SPI replace $A$ by $B$ ). The indices $r$ and $i$ denote real and imaginary parts, respectively. As an aside we mention that a linear variation of $\Omega_{A}$ with $|A|^{2}$ holds for SPI also significantly further away from onset than in Fig. 2]

Motivated by Fig. 2 we also discard quintic contributions to the modulus and to the frequency of the RIB state. This is enforced by making in the terms $f|B|^{4} A$ and $f|A|^{4} B$ that appear on general grounds in (4a) and (4b), respectively, the special choice $f=-e$. Then the RIB solution is characterized by $|A|^{2}=|B|^{2}=\mu /\left(-b_{r}-c_{r}\right)$ and $\Omega_{A}=\Omega_{B}=$ $-a_{i} \mu-\left(b_{i}+c_{i}\right)|A|^{2}$.

The CR-SPI solution with finite $A \neq B$ exists only for nonzero coupling $e$. It is most conveniently found and expressed in terms of the combined order parameters

$$
\begin{array}{lll}
S=\left(|A|^{2}+|B|^{2}\right) / 2 & , & D=\left(|A|^{2}-|B|^{2}\right) / 2 \\
\Omega_{S}=\left(\Omega_{A}+\Omega_{B}\right) / 2 & , & \Omega_{D}=\left(\Omega_{A}-\Omega_{B}\right) / 2 .
\end{array}
$$

Then the radial velocity field (3) of the CR-SPI at mid-gap can be written into the form $u=$ $\left[|A| e^{i\left(k z-\Omega_{D} t\right)}+|B| e^{-i\left(k z-\Omega_{D} t\right)}\right] e^{i\left(M \varphi-\omega^{0} t-\Omega_{S} t\right)}+c . c$. . It shows the aforementioned rotation frequency $\omega_{S}=\omega^{0}+\Omega_{S}$ and the axial propagation of the deformation that is governed by the frequency $\omega_{D}=\Omega_{D}$. 
The CR-SPI solution of (4) reads

$$
\begin{array}{r}
S=\left(c_{r}-b_{r}\right) /\left(2 e_{r}\right) \quad, \quad D^{2}=\left[\mu+\left(b_{r}+c_{r}\right) S\right] /\left(2 e_{r}\right) \\
-\Omega_{S}=a_{i} \mu+\left(b_{i}+c_{i}\right) S-2 e_{i} D^{2} \quad, \quad-\Omega_{D}=\left(b_{i}-c_{i}+2 e_{i} S\right) D .
\end{array}
$$

Note that $S$ is constant in this CR-SPI. $D$ grows via a pitchfork bifurcation at $\mu_{2}=-\left(b_{r}+\right.$ $\left.c_{r}\right) S$ out of the RIB $(D=0)$ and connects at $\mu_{1}=-2 b_{r} S$ with $D=|A|^{2} / 2=S$ with the L-SPI and with $D=-|B|^{2} / 2=-S$ with the R-SPI.

In Figs. 2 - 4 we compare bifurcation diagrams of SPI, RIB, and CR-SPI solutions resulting from the amplitude equations [11] (thin lines) with those from the full numerical simulations (symbols). For the sake of presentational clarity the stability of the former is not indicated in these figures. But stability analyses of the three vortex states in question confirms that the CR-SPI solution (6) transfers stability between the RIB and the SPI states as indicated in Fig. 5

We should like to mention that keeping the discarded quintic terms by setting $d \neq 0$ and $f \neq-e$ slightly improves the agreement between the solutions of the Navier-Stokes equations and the amplitude equation approximation without changing the bifurcation topology and the stability behavior of the latter. However, then the CR-SPI solution would still have $S=$ const. To obtain deviations from this behavior as displayed, e.g., by the triangles of Fig. 4(a) one needs higher orders in eqs. (4).

So, the model (41) is a minimal one in the sense that the quintic terms that are necessary to ensure the existence of $A \neq B$ solutions enter only into the coupling between $\mathrm{R}$ - and L-SPI modes but not into the SPI and RIB solutions. Note furthermore that the coupled amplitude equations (41) apply equally well to other systems with two distinct $A$ and $B$ solutions - be they stationary or oscillatory — that are related to each other by analogous (symmetry) requirements with similar nonlinear couplings. Thus, bifurcation diagrams in which the pure $A$, the pure $B$, and the $A=B$ solutions are connected with a stability transfer by a $A \neq B$ solution branch could also arise in these systems with a form that would be (topologically) similar to that of Fig. 5 .

This work was supported by the Deutsche Forschungsgemeinschaft. 
[1] M. C. Cross and P. C. Hohenberg, Rev. Mod. Phys. 65, 851 (1993).

[2] R. Tagg, Nonlinear Science Today 4, 1 (1994).

[3] P. Chossat and G. Iooss, The Couette-Taylor Problem, (Springer, Berlin, 1994).

[4] Stable RIBs were found [5] in a long system of aspect ratio $\Gamma=36$ with radius ratio $\eta=0.727$ where theory [3, 6] and numerical simulations [5] predicted a subcritical transition. However, no CR-SPI were obseved. RIBs in end-plate-dominated short systems $(\Gamma<10, \eta=1 / 2)$ were recently reported [7] to come with two different symmetries [8].

[5] R. Tagg, W. S. Edwards, H. L. Swinney, and P. S. Marcus, Phys. Rev. A 39, 3734 (1989).

[6] Y. Demay and G. Iooss, J. Mec. Theor. Appl., Spec. Suppl., 193 (1984).

[7] J. Langenberg, G. Pfister, and J. Abshagen, Phys. Rev. E 68, 056308 (2003); Phys. of Fluids, 16, 2757 (2004).

[8] E. Knobloch and R. Pierce, in Ordered and turbulent patterns in Taylor-Couette Flow, ed. C. D. Andereck and F. Hayot, (Plenum Press, NY, 1992), p. 83.

[9] Ch. Hoffmann, M. Lücke, and A. Pinter, Phys. Rev. E 69, 056309 (2004).

[10] S. Fauve, S. Douady, and O. Thual, J. Phys. II 1, 311 (1991) show that a non-potential linear coupling which is forbidden here can cause a pitchfork drift bifurcation of parametrically excited surface SWs into reflection symmetry breaking traveling states with $A \neq B$.

[11] We have determined the coefficients as follows: a linear analysis of the flow dynamics gave $\omega^{0}=150.9$ (117.0) and $a_{i}=45.13(15.97)$ for $R_{1}=240\left(R_{1}=200\right)$. Then $b_{r}$ and $b_{i}$ were obtained by fitting $|A|^{2}$ and $\Omega_{A}$, respectively, of the nonlinear numerical SPI solution. Thereafter $c_{r}$ and $c_{i}$ were fixed analogously with the numerical RIB solutions. Finally, $e$ was determined with the CR-SPI. The results are $100 b=-0.245+i 83.54(-0.659+i 132.8)$, $100 c=-1.06-i 554(-2.517-i 383.9), 100 e=-3.251+i 118.4(-1.976+i 89.2)$.

[12] Unstable SPI solutions were obtained by suppressing the $m=0$-mode of $u$. This stabilizes the SPI against the transition to CR-SPI and RIB states which have finite $m=0$ - content. The unstable RIB solution was stabilized by imposing the $z \rightarrow-z$ symmetry of this state thereby suppressing SPI and CR-SPI solutions. 
(a) L-SPI
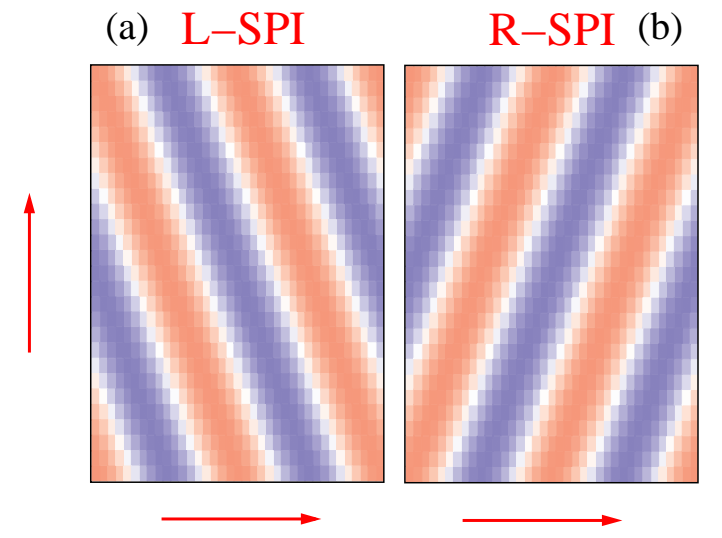

(c)

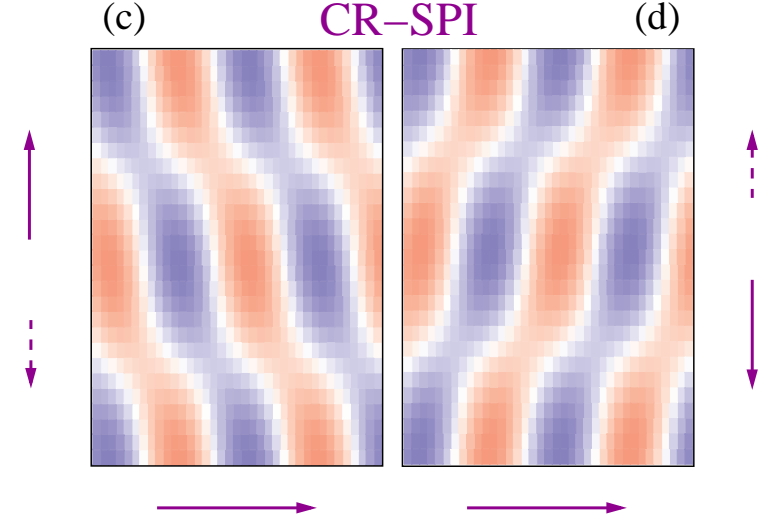

(e) RIB

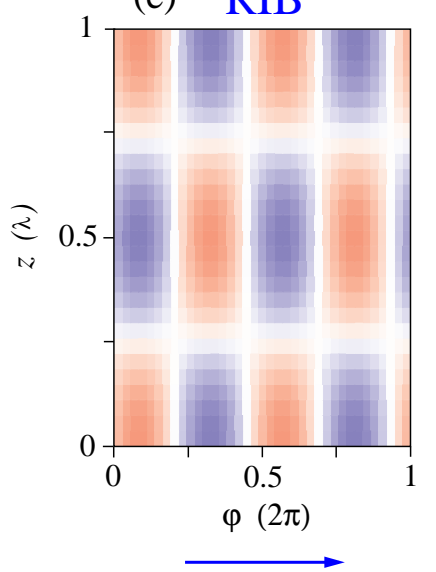

FIG. 1: (Color online) Snapshots of the radial flow $u$ in the $\varphi-z$-plane of the 'unrolled' midgap cylindrical surface. White identifies the nodes. Light (dark) grey [red (blue) in the electronic version] marks radial out-flow (in-flow). Arrows indicates how the phase moves. For the CR-SPI in (c) [(d)] the undulated pattern moves upwards [downwards] (full arrows) while the undulation itself propagates slowly downwards [upwards] (dashed arrows). Parameters are $R_{2}=-605.2(a, b),-604.725(c, d),-604.5(e)$ and $R_{1}=240$. 


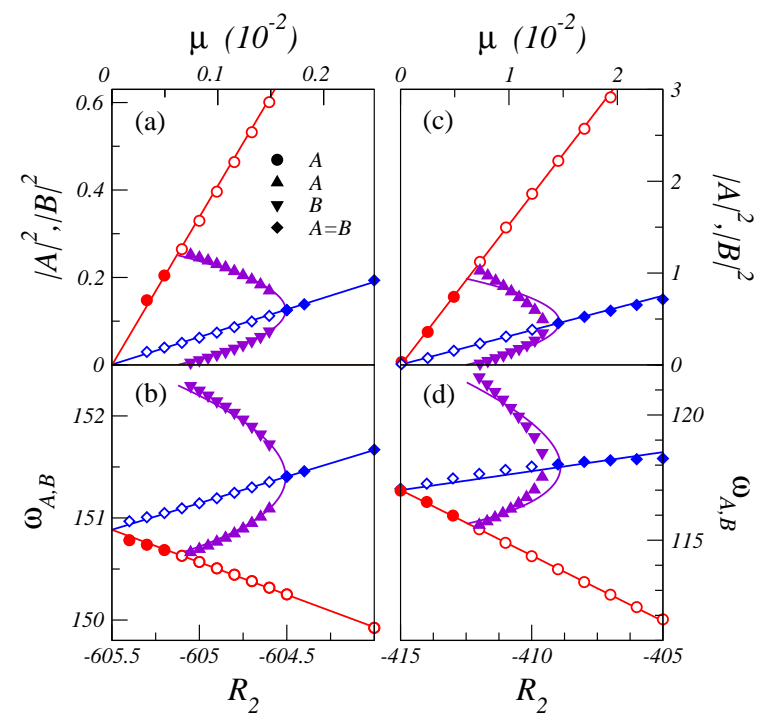

FIG. 2: (Color online) Bifurcation diagrams of SPI (red circles), RIB (blue diamonds), and CRSPI (purple triangles) obtained from numerical solutions of the full Navier-Stokes equations for $R_{1}=240(\mathrm{a}, \mathrm{b})$ and $R_{1}=200(\mathrm{c}, \mathrm{d})$ as functions of $R_{2}$ and $\mu$. Shown are the squared mode amplitudes $|A|^{2},|B|^{2}$ and frequencies $\omega_{A}, \omega_{B}$ of the dominant modes $u_{2,1}(t), u_{2,-1}(t)$ (2)), respectively, in the expansion (11) of the radial velocity field $u$ at mid-gap. Full (open) symbols denote stable (unstable) solutions [12]. Lines show amplitude-equation approximations. Here a CR-SPI with $|A|>|B|, \omega_{A}<\omega_{B}$ transfers stability from an L-SPI $(A \neq 0, B=0)$ to the RIB state $(A=B)$. The analogous diagram with an R-SPI is obtained by interchanging $A$ and $B$. 


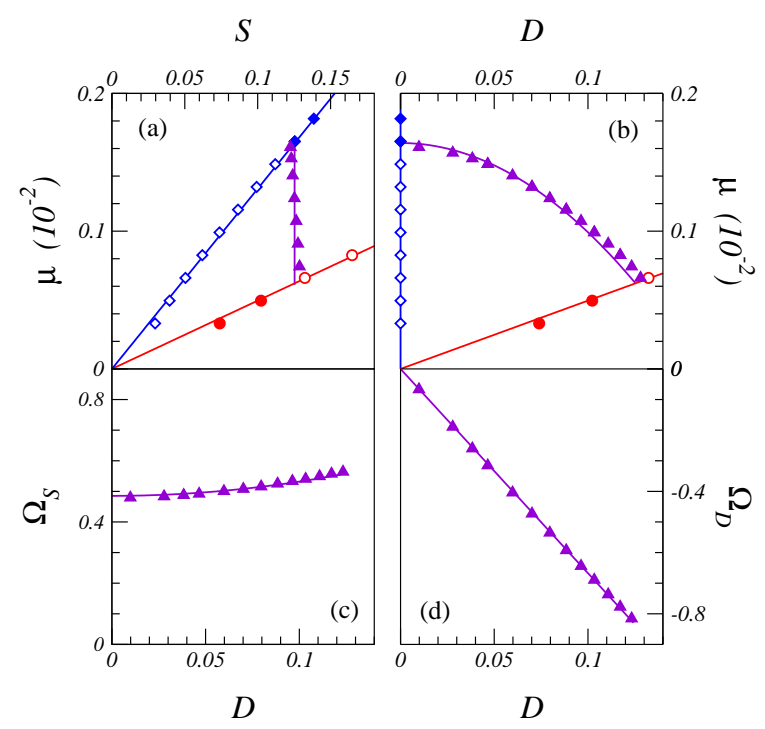

FIG. 3: (Color online) Bifurcation diagrams of SPI (red circles), RIB (blue diamonds), and CR-SPI (purple triangles) obtained from numerical solutions of the full Navier-Stokes equations for $R_{1}=$ 240 using the order parameters (5): Relation between $\mu$ and $S$ (a) and $D$ (b), respectively; CR-SPI frequencies $\Omega_{S}=\left(\Omega_{A}+\Omega_{B}\right) / 2=\left(\omega_{A}+\omega_{B}\right) / 2-\omega^{0}(\mathrm{c})$ and $\Omega_{D}=\left(\Omega_{A}-\Omega_{B}\right) / 2=\left(\omega_{A}-\omega_{B}\right) / 2=\omega_{D}$ (d), respectively, versus $D$. Full (open) symbols denote stable (unstable) solutions 12]. Lines show amplitude-equation approximations. Here a CR-SPI $\left(|A|>|B|, D>0\right.$ and $\left.\Omega_{A}<\Omega_{B}, \Omega_{D}<0\right)$ transfers stability from an L-SPI $(B=0$, i.e., $D=S)$ to the RIB state $(A=B$, i.e., $D=0)$. 


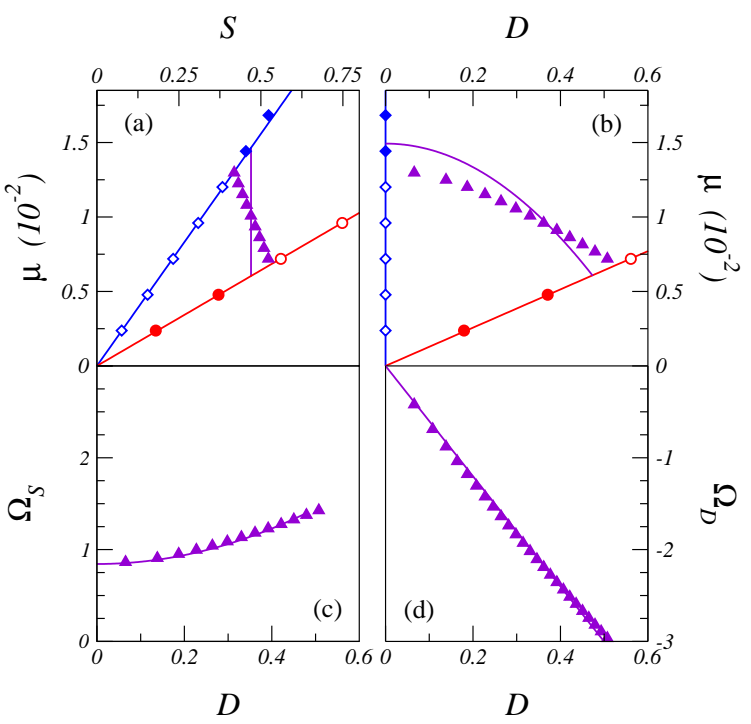

FIG. 4: (Color online) Bifurcation diagrams for $R_{1}=200$ as in Fig. 3 . 
(a)

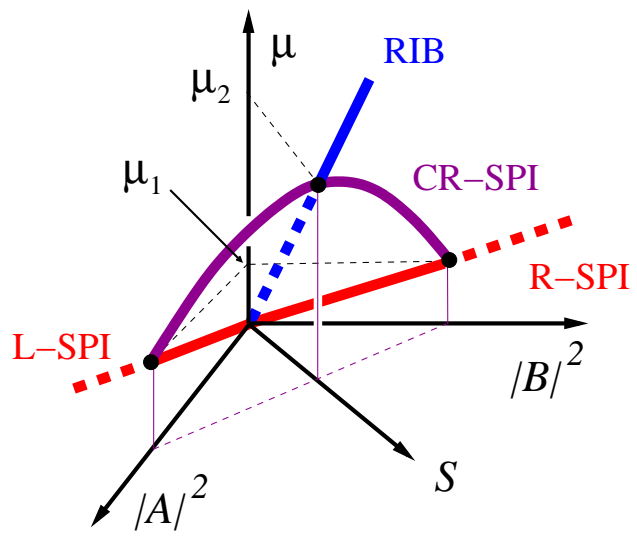

(b)

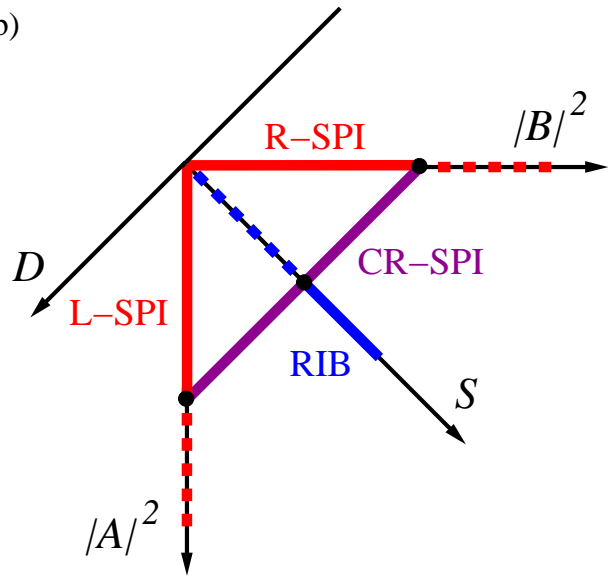

FIG. 5: (Color online) Bifurcation diagrams of SPI, RIB, and CR-SPI resulting from the amplitudeequation approximation. (a) 3D plot in $|A|^{2}-|B|^{2}-\mu$-space; (b) projection onto the $|A|^{2}-|B|^{2}$ plane or the $D-S$-plane, respectively. Full (dashed) lines denote stable (unstable) solutions. The CR-SPI connects with constant $S$ the two SPI solution branches to the RIB thereby transferring stability from the former to the latter. 